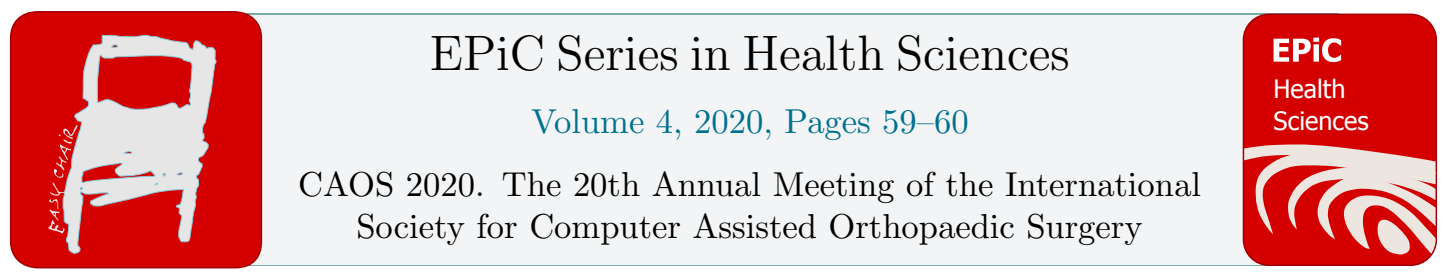

\title{
Predictability of Implant Size in Robotic Surgery for Total Knee and Total Hip Replacement Arthroplasty
}

\author{
Kamal Deep, Frederic Picard, Shoaib Iqbal \\ Nuffield Hospital and Golden Jubilee National Hospital Glasgow UK \\ mrkdeep@gmail.com
}

\begin{abstract}
Accuracy of implantation using computer assisted navigation and robotic total knee replacement arthroplasty (TKR) and total hip replacement (THR) has been proven. Templating the pre-operative radiographs has become standard. This gives an insight into the pre op planning and the sizes most likely to be used. This helps to reduce the inventory for storage of implants and cost. While the templating of radiographs has been helpful, implant sizes prediction remains less than desirable. Aim of present study was to look at the predictability of implant sizes in CT planning for robotic surgery. 30 MAKO robotic joint replacements were performed (15TKR/15THR) with pre op CT scans for implant size. For TKR, the sizes used were mean 5 in femur and tibia (SD1 and range 3-7). In tibia, size used was same as predicted. In the femur in two cases the size was reduced by one to balance the gaps. Insert thickness was increased by one size in 4 cases. For THR, the acetabular cup, femur, head diameter and offset were predicted $100 \%$. The neck length had to be changed in some cases by up to two sizes. for balance/stability. In conclusion the CT scan pre-operative planning for MAKO robotic knee joint replacement can predict $100 \%$ times the size of tibia, within one size of femur and insert for TKR. These figures are better than published predictability of templates of plain radiographs where implant size was predicted $42 \%$ for femoral and $37 \%$ acetabular components while $87 \%$ of the femoral components and $78 \%$ of the acetabular cups were accurate within one size up and down.
\end{abstract}

Introduction: The accuracy of implantation using computer assisted navigation $(1,2,3)$ and robotic total knee replacement arthroplasty (TKR) and total hip replacement (THR) has been proven and accepted $(4,5,6)$. Templating the pre-operative radiographs has become standard in many modern units of joint arthroplasty (7). This gives an insight into the pre op planning and the sizes that will most likely be used during the surgery. This helps to reduce the inventory on the shelf for storage of implants as well as reduces the total numbers required as you can order the required sizes only instead of having the full inventory which costs the company as well as the hospitals, needing a lot more manpower and economic resources in the process. While the templating of radiographs has been helpful it has not led to complete belief in the system and predictability of the implant sizes remains less than desirable. 
Aim: Aim of present study was to look at the predictability of implant sizes in CT planning for robotic surgery. Method: 30 MAKO robotic joint replacements were performed including 15 total knee replacements and 15 total hip replacements. The pre op planning was done on CT scans. This gave the sizes of the implants. Results and Discussion: For TKR, the sizes used were mean 5 in femur as well as tibia with SD of 1 and range 3-7. In tibia the size used was always the same as predicted on the pre-operative planning of the CT scans. In the femur only in two cases the femoral size was reduced by one size to adjust for the soft tissue gaps as flexion gap was tight to begin with, which is unpredictable on the CT scans. The predictability of the insert thickness was not as good and had to be increased by one size in 4 cases. For the hips, the acetabular as well the femoral sizes were planned on the CT scan. For the acetabular cup the sizes were predicted $100 \%$ times. For the femur size as well the offset the sizes were predicted $100 \%$ times. The head diameter was used, as had been planned $100 \%$ times. The neck length had to be changed due to the soft tissue and clinical feel in many cases either changing 4 or $8 \mathrm{~mm}$ length to the pre-planned case, in order to maximise per operative balance and stability. In conclusion the $\mathrm{CT}$ scan pre-operative planning for MAKO robotic knee joint replacement can predict $100 \%$ times the size of tibia, within one size of femur and insert. CT scan pre-operative planning for MAKO robotic hip joint replacement can predict $100 \%$ times the size of cup, size of femur, size of liner, size of head, and within two sizes of the neck length. These figures are better than published predictability of templates of the plain radiographs. (7). In the 2D digital templating the implant size was predicted exactly in $42 \%$ for the femoral and in $37 \%$ for the acetabular component. $87 \%$ of the femoral components and $78 \%$ of the acetabular cups were accurate within one size (7). The weakness of the study could be that the numbers are small and when it goes to hundreds there may be variations, but the authors predict there may be variation of up to one size of the main implants. The poly insert and neck length sizes may still vary by two sizes. Hence the CT scan pre planning for MAKO will help in managing the implant inventory better and may reduce the delays in getting the implant to the operating room as we already know which size should be available for opening immediately when it is required. Authors believe that such high accuracy is due to the $\mathrm{CT}$ based $3 \mathrm{D}$ planning as well as robotic based preparation of the bones. However, authors do not recommend that other sizes should not be available nearby if needed.

\section{References}

1. Liu Z, Gao Y, Cai L (2015) Imageless navigation versus traditional method in total hip arthroplasty: a meta-analysis. Int J Surg 21, 122-127.

2. Deep K, Shankar S \& Mahendra A (2017) Computer assisted navigation in total knee and hip arthroplasty. SICOT J, 3:50.

3. Hetaimish BM, Khan MM, Simunovic N, Al-Harbi HH, Bhandari M, Zalzal PK (2012) Metaanalysis of navigation vs conventional total knee arthroplasty. J Arthroplasty 27(6), 1177-1182.

4. Bargar WL. Robots in orthopaedic surgery: past, present, and future. Clin Orthop Relat Res 2007;463:31-6.

5. Domb BG, El Bitar YF, Sadik AY, et al. Comparison of robotic-assisted and conventional acetabular cup placement in THA: a matched-pair controlled study. Clin Orthop Relat Res 2014;472:329-36.

6. Nakamura N, Sugano N, Nishii T, et al. A comparison between robotic-assisted and manual implantation of cementless total hip arthroplasty. Clin Orthop Relat Res 2010;468:1072-81.

7. Holzer, L.A., Scholler, G., Wagner, S. et al. Arch Orthop Trauma Surg (2019) 139: 263. https://doi.org/10.1007/s00402-018-3080-0 